\title{
Time-reversible molecular dynamics algorithms with bond constraints
}

Toxværd, Søren; Heilmann, Ole; Ingebrigtsen, Trond; Schrøder, Thomas; Dyre, J. C.

Published in:

Journal of Chemical Physics

DOI:

10.1063/1.3194785

Publication date:

2009

Document Version

Publisher's PDF, also known as Version of record

Citation for published version (APA):

Toxværd, S., Heilmann, O., Ingebrigtsen, T., Schrøder, T., \& Dyre, J. C. (2009). Time-reversible molecular dynamics algorithms with bond constraints. Journal of Chemical Physics, 131(6), 064102-1 til 064102-9. https://doi.org/10.1063/1.3194785

\section{General rights}

Copyright and moral rights for the publications made accessible in the public portal are retained by the authors and/or other copyright owners and it is a condition of accessing publications that users recognise and abide by the legal requirements associated with these rights.

- Users may download and print one copy of any publication from the public portal for the purpose of private study or research.

- You may not further distribute the material or use it for any profit-making activity or commercial gain.

- You may freely distribute the URL identifying the publication in the public portal.

\section{Take down policy}

If you believe that this document breaches copyright please contact rucforsk@kb.dk providing details, and we will remove access to the work immediately and investigate your claim. 


\title{
Time-reversible molecular dynamics algorithms with bond constraints
}

\author{
Søren Toxvaerd, ${ }^{\text {a) }}$ Ole J. Heilmann, Trond Ingebrigtsen, Thomas B. Schrøder, and \\ Jeppe C. Dyre \\ Department of Sciences, DNRF Centre "Glass and Time," IMFUFA, Roskilde University, \\ P.O. Box 260, DK-4000 Roskilde, Denmark
}

(Received 2 April 2009; accepted 14 July 2009; published online 10 August 2009)

\begin{abstract}
Time-reversible molecular dynamics algorithms with bond constraints are derived. The algorithms are stable with and without a thermostat and in double precision as well as in single-precision arithmetic. Time reversibility is achieved by applying a central-difference expression for the velocities in the expression for Gauss' principle of least constraint. The imposed time symmetry results in a quadratic expression for the Lagrange multiplier. For a system of complex molecules with connected constraints the corresponding set of coupled quadratic equations is easily solved by a consecutive iteration scheme. The algorithms were tested on two models. One is a dumbbell model of Toluene, the other system consists of molecules with four connected constraints forming a triangle and a branch point of constraints. The equilibrium particle distributions and the mean-square particle displacements for the dumbbell model were compared to the corresponding functions obtained by GROMACS. The agreement is perfect within statistical error. () 2009 American Institute of Physics. [DOI: 10.1063/1.3194785]
\end{abstract}

\section{INTRODUCTION}

Holonomic constraints are used in many contexts of complex dynamics. Most commonly, perhaps, are holonomic constraints used in molecular dynamics (MD) simulations of molecular systems in order to eliminate the fast modes. In this context the advantage of applying constraints is that one can use larger time steps. Another example is the use of constraints in order to ensure a certain conformation or for calculating reaction rates. Since Ryckaert et al. ${ }^{1}$ developed the SHAKE algorithm, a series of refined numerical algorithms for holonomic constrained MD has been proposed. ${ }^{2-9}$ Whereas SHAKE ensures the constraints by occasional rescaling of the coordinates, the latter algorithms are based on Gauss's principle of least constraint. ${ }^{10}$ The derived expressions depend linearly on the Lagrange multipliers and the algorithms used for discrete MD are not time symmetrical. The algorithms do not conserve energy nor the bond lengths. For this reason the simulations are performed with a thermostat (NVT-MD) and a series of "penalty" procedures has been developed. ${ }^{1-3,5,6}$ The derived algorithms are, however, robust and implemented in many program packages that are widely used for simulating complex molecular systems.

In the present article we derive simple reversible algorithms for the time-discrete MD with bond constraints with and without a thermostat. The algorithms conserve energy and are stable. The algorithms are derived from Sec. II. In Sec. III we describe how to solve the coupled quadratic equations for connected constraints in complex molecules. The algorithms are tested on a Lennard-Jones (LJ) dimer (united atom) model for toluene (model I) and a system of more complex molecules (model II) in Sec. IV. The results are summarized and discussed in Sec. V.

${ }^{a)}$ Electronic mail: st@ruc.dk.

\section{TIME-REVERSIBLE DISCRETE DYNAMICS WITH CONSTRAINTS}

\section{A. Holonomic constraints}

For simplicity we consider a system of $N$ dimers with masses $m_{A}$ and $m_{B}$ with constant distance $d$ between the two subunits. The dynamics of the system of dimers with the holonomic constraints is given by Gauss's principle of least constraint. ${ }^{2}$ The holonomic constraint between one of the dimers with positions $\mathbf{r}_{1}(t)$ and $\mathbf{r}_{2}(t)$ at time $t$ is

$$
\mathbf{r}_{12}^{2}(t)=d^{2},
$$

where $\mathbf{r}_{12}=\mathbf{r}_{1}-\mathbf{r}_{2}$. Differentiating this equation gives

$$
\mathbf{r}_{12}(t) \cdot \mathbf{v}_{12}(t)=0,
$$

which expresses that the velocity $\mathbf{v}_{12}(t)$ along the bond direction is zero. A second time differentiation gives

$$
\mathbf{r}_{12}(t) \cdot \mathbf{a}_{12}(t)+\mathbf{v}_{12}^{2}(t)=0,
$$

which gives the constraint for the acceleration $\mathbf{a}_{12}(t)$. Let the subunit at $\mathbf{r}_{1}(t)$ have the mass $m_{A}$ and the subunit at $\mathbf{r}_{2}(t)$ the mass $m_{B}$. Expressing the constraint forces as $\pm \lambda \mathbf{r}_{12}(t)$, the Hamilton equations for the two subunits are ${ }^{2}$

$$
\begin{aligned}
& m_{A} \frac{d \mathbf{r}_{1}}{d t}=\mathbf{p}_{1}, \\
& \frac{d \mathbf{p}_{1}}{d t}=\mathbf{f}_{1}+\lambda \mathbf{r}_{12}, \\
& m_{B} \frac{d \mathbf{r}_{2}}{d t}=\mathbf{p}_{2},
\end{aligned}
$$




$$
\frac{d \mathbf{p}_{2}}{d t}=\mathbf{f}_{2}-\lambda \mathbf{r}_{12} .
$$

The forces on a mass unit $i$ can be separated into intra- and intermolecular forces; for a dimer $(i, i+1)$, one has

$$
\begin{aligned}
& \mathbf{f}_{i}=\mathbf{f}_{i, i+1}+\Sigma_{j \neq i+1} \mathbf{f}_{i, j}, \\
& \mathbf{f}_{i+1}=-\mathbf{f}_{i, i+1}+\Sigma_{j \neq i} \mathbf{f}_{i+1, j} .
\end{aligned}
$$

By substitution of the equations for the dynamics into Eq. (3) one obtains the analytic expression for $\lambda(t)$ (Ref. 2),

$$
\lambda(t)=-\mu \frac{\mathbf{r}_{12}(t) \cdot \hat{\mathbf{f}}_{12}(t)+\mathbf{v}_{12}^{2}(t)}{\mathbf{r}_{12}^{2}(t)},
$$

where the intramolecular forces in Eqs. (8) and (9) do not contribute to $\hat{\mathbf{f}}_{12}(t)$,

$$
\hat{\mathbf{f}}_{12}(t)=\mathbf{f}_{1}(t) / m_{A}-\mathbf{f}_{2}(t) / m_{B},
$$

and the reduced mass $\mu$ as usual is defined by

$$
\mu=\frac{m_{A} m_{B}}{m_{A}+m_{B}} .
$$

In MD simulations the discrete time dynamics is usually performed by a simple central-difference algorithm. This timesymmetrical algorithm can be expressed in several ways, e.g., by Verlet, ${ }^{1}$

$$
\mathbf{r}_{i}(t+h)=2 \mathbf{r}_{i}(t)-\mathbf{r}_{i}(t-h)+h^{2} \mathbf{f}_{i}(t),
$$

from which a new set of positions at time $t+h$ is obtained from the two previous sets of positions at time $t$ and $t-h$ and the forces at time $t$. Another formulation of the algorithm is the "leap frog algorithm" (LFA) (Ref. 12) and there are several other formulations of the central-difference algorithm. The algorithm has two significant qualities: It is time reversible and the trajectories are obtained directly from the forces in Eq. (13) without any dependence on the velocities. In fact there exists no unique expression for the velocity at the time $(t)$, where the forces are calculated from the positions. ${ }^{13} \mathrm{In}$ the LFA one has a corresponding central-difference expression for the velocity at the $n$th time step $(n-1 / 2) h$, and the updating of positions is performed in two steps, first by updating the velocity to time $(n+1 / 2) h$ and then the positions to time $(n+1) h$. The Verlet algorithm is the centraldifference algorithm for Newton's formulation of classical dynamics; the LFA is the corresponding central-difference algorithm for Hamilton's formulation.

The above implementation of the holonomic constraints leaves two fundamental problems: Since the velocities do not enter into the dynamics, any roundoff error in the dynamics will lead to a drift in the velocities which makes the algorithms numerically unstable. Another problem is to ensure time reversibility. In the LFA formulation a natural time symmetric and central-difference expression for the velocity difference $\mathbf{v}_{12}^{2}(t)$ in Eq. (10) is

$$
\mathbf{v}_{12}^{2}(t)=\frac{\mathbf{v}_{12}^{2}(t+h / 2)+\mathbf{v}_{12}^{2}(t-h / 2)}{2},
$$

where the LFA corresponding to Eqs. (4)-(7) gives

$$
\mathbf{v}_{12}(t+h / 2)=\mathbf{v}_{12}(t-h / 2)+h \hat{\mathbf{f}}_{12}(t)+h \lambda(t) \mathbf{r}_{12}(t) / \mu .
$$

Substituting Eq. (15) into Eq. (14) one obtains from Eq. (10) the following second-order equation in $\lambda$ :

$$
\alpha \lambda^{2}+\beta \lambda+\gamma=0,
$$

where

$$
\begin{aligned}
& \alpha=h^{2} / 2 \mu, \\
& \beta=1+\frac{h \mathbf{r}_{12}(t) \cdot\left(\mathbf{v}_{12}(t-h / 2)+h \hat{\mathbf{f}}_{12}(t)\right)}{\mathbf{r}_{12}^{2}(t)},
\end{aligned}
$$

Equation (16) has two roots, but only the root

$$
\lambda=\frac{-\beta+\sqrt{\beta^{2}-4 \alpha \gamma}}{2 \alpha}
$$

is consistent with Eq. (10) as $h \rightarrow 0$ (the negative root diverges as $O\left(h^{-2}\right)$ and the discriminant is negative after one time step, i.e., the constrained discrete dynamics has no real solution in this case). The linear expression Eq. (10) for the constraint is changed to a quadratic expression due to the requirement of time symmetry and it complicates the dynamics for connected constraints with coupled quadratic equations. The coupled quadratic equations can, however, be solved by simple "consecutive iterations" (see Sec. III).

\section{B. Time-reversible discrete dynamics with bond constraints and a thermostat}

MD can be simulated with a time reversible NoséHoover thermostat. ${ }^{12}$ The dynamics for the Nosé-Hoover thermostat is given by a coupling of the momenta to the thermostat. The constrained Hamilton equations with bond and temperature constraints are

$$
\begin{aligned}
& m_{A} \frac{d \mathbf{r}_{1}}{d t}=\mathbf{p}_{1}, \\
& \frac{d \mathbf{p}_{1}}{d t}=\mathbf{f}_{1}+\lambda \mathbf{r}_{12}-\xi \mathbf{p}_{1},
\end{aligned}
$$




$$
\begin{aligned}
& m_{B} \frac{d \mathbf{r}_{2}}{d t}=\mathbf{p}_{2}, \\
& \frac{d \mathbf{p}_{2}}{d t}=\mathbf{f}_{2}-\lambda \mathbf{r}_{12}-\xi \mathbf{p}_{2}, \\
& \frac{d \xi(t)}{d t}=a\left(k(t)-k_{0}\right),
\end{aligned}
$$

where $\xi(t)$ is a "friction" with a coupling constant/strength $a$ to the thermostat, $k(t)$ is the kinetic energy per degree of freedom in the system, and $k_{0}(T)$ is the kinetic target energy. The additional friction term enters into the equation for $\lambda$ and Eq. (10) is modified to

$$
\lambda(t)=-\mu \frac{\mathbf{r}_{12}(t) \cdot\left(\hat{\mathbf{f}}_{12}(t)-\xi(t) \mathbf{v}_{12}(t)\right)+\mathbf{v}_{12}^{2}(t)}{\mathbf{r}_{12}^{2}(t)}
$$

In a discrete LFA formulation of the NVT-MD one knows the positions, forces, and friction $\xi$ at time $t$ and the velocities at time $t-h / 2 .^{12}$ Time reversibility of the holonomic NVT-dynamics is ensured in the same manner as with bond constraints by a central mean

$$
\mathbf{v}_{12}(t)=\frac{\mathbf{v}_{12}(t+h / 2)+\mathbf{v}_{12}(t-h / 2)}{2} .
$$

The new term in the LFA dynamics ${ }^{12}$ modifies the expression Eq. (15) for $\mathbf{v}_{12}(t+h / 2)$ to

$$
\begin{aligned}
\mathbf{v}_{12}(t+h / 2)= & \frac{1}{1+\xi h / 2}\left(\mathbf{v}_{12}(t-h / 2)(1-\xi h / 2)+h \hat{\mathbf{f}}_{12}(t)\right. \\
& \left.+h \lambda(t) \mathbf{r}_{12}(t) / \mu\right)
\end{aligned}
$$

and gives rise to new terms and modifications of Eqs. (17)-(20). With the abbreviations

$$
\begin{aligned}
& \xi_{1}=1+\xi h / 2, \\
& \xi_{2}=1-\xi h / 2, \\
& \xi_{3}=1+(\xi h)^{2} / 4,
\end{aligned}
$$

and

$$
\hat{\mathbf{f}}_{12}^{\prime}=\hat{\mathbf{f}}_{12}(t)\left(1-\xi h / 2 \xi_{1}\right)-\xi / \xi_{1} \mathbf{v}_{12}(t-h / 2),
$$

the modified expressions are

$$
\begin{aligned}
& \alpha=h^{2} /\left(2 \mu \xi_{1}^{2}\right), \\
& \beta=1-\frac{\xi h}{2 \xi_{1}}+\frac{h \mathbf{r}_{12}(t) \cdot\left(\xi_{2} \mathbf{v}_{12}(t-h / 2)+h \hat{\mathbf{f}}_{12}(t)\right)}{\xi_{1}^{2} \mathbf{r}_{12}^{2}(t)},
\end{aligned}
$$

The NVT-MD dynamics with bond constraints is performed as the corresponding $N V E$-MD $(\xi=0)$ dynamics using Eqs. (33)-(35) for both ensembles without further complications ("method 0").

\section{Improved and numerically stable algorithms}

So far we have ensured the time symmetry in the equations and the derived algorithm will formally conserve the bond length in the case of no roundoff errors (see derivation below). From a numerical point of view, however, the algorithm is unstable and roundoff errors will accumulate, and is confirmed by the simulations. Since the velocities do not influence the trajectories obtained by the equivalent centraldifference algorithm [Verlet algorithm Eq. (13)], it indicates that a drift in the bond distances will not be corrected by the method in Secs. II A and II B. This fact can as well be seen from the derivation below.

From the next step in the LFA

$$
\mathbf{r}_{i}(t+h)=\mathbf{r}_{i}(t)+h \mathbf{v}_{i}(t+h / 2),
$$

one obtains by squaring the corresponding expression for $\mathbf{r}_{12}(t+h)$

$$
\begin{aligned}
\mathbf{r}_{12}(t+h)^{2}-\mathbf{r}_{12}(t)^{2}= & h^{2} \mathbf{v}_{12}(t+h / 2)^{2} \\
& +2 h \mathbf{v}_{12}(t+h / 2) \cdot \mathbf{r}_{12}(t) .
\end{aligned}
$$

If Eq. (15) is substituted into this and the terms are arranged after powers of $\lambda$ one gets using Eqs. (17)-(19)

$$
\begin{aligned}
\mathbf{r}_{12}(t+h)^{2}-\mathbf{r}_{12}(t)^{2}= & \left(2 h^{2} \mathbf{r}_{12}(t)^{2} / \mu\right)\left[\alpha \lambda^{2}+\beta \lambda+\gamma\right] \\
& +2 h \mathbf{v}_{12}(t-h / 2) \cdot \mathbf{r}_{12}(t) \\
& -h^{2} \mathbf{v}_{12}(t-h / 2)^{2} .
\end{aligned}
$$

Similar to Eq. (36) we have for the previous LFA time step

$$
\mathbf{r}_{12}(t-h)=\mathbf{r}_{12}(t)-h \mathbf{v}_{12}(t-h / 2),
$$

which yields

$$
\begin{aligned}
\mathbf{r}_{12}(t-h)^{2}-\mathbf{r}_{12}(t)^{2}= & h^{2} \mathbf{v}_{12}(t-h / 2)^{2} \\
& -2 h \mathbf{v}_{12}(t-h / 2) \cdot \mathbf{r}_{12}(t),
\end{aligned}
$$

and from Eqs. (40) and (38)

$$
\begin{aligned}
\mathbf{r}_{12}(t+h)^{2}-\mathbf{r}_{12}(t)^{2}= & \left(2 h^{2} \mathbf{r}_{12}(t)^{2} / \mu\right)\left[\alpha \lambda^{2}+\beta \lambda+\gamma\right] \\
& +\mathbf{r}_{12}(t)^{2}-\mathbf{r}_{12}(t-h)^{2} .
\end{aligned}
$$

If one uses Eq. (16) to determine $\lambda$, Eq. (41) is reduced to 


$$
\mathbf{r}_{12}(t+h)^{2}-\mathbf{r}_{12}(t)^{2}=\mathbf{r}_{12}(t)^{2}-\mathbf{r}_{12}(t-h)^{2}+\boldsymbol{\epsilon}_{n},
$$

where $\epsilon_{n}$ is the error introduced at the $n$th step. This equation shows that a drift in the bond lengths (and an error in the start configurations), as expected, is carried over to the next step of the simulation. (A change in the bond length by a drift is given by $r_{12}(t)-r_{12}(t-h)$ and Eq. (42) shows that a drift is carried over to the next step as $\left(\mathbf{r}_{12}(t)+\mathbf{r}_{12}(t\right.$ $-h)) \cdot\left(\mathbf{r}_{12}(t)-\mathbf{r}_{12}(t-h)\right) \approx 2 d\left(r_{12}(t)-r_{12}(t-h)\right)$.) But even if the simulation starts from a perfect state, any error will increase linearly as the integration progresses. If one assumes that the errors introduced at different steps of the method are independently distributed stochastic variables, the variance of the total error increases with $n^{3}$, where $n$ is the number steps of the integration algorithm. If the individual errors have a bias, the total bias will increase with $n^{2}$ [actually, our calculations indicate that the roundoff errors are not uncorrelated but that does not change these general conclusions about the instability of Eq. (42)].

If instead of Eq. (16) one had used Eq. (10) with $\mathbf{v}_{12}(t$ $-h / 2)$ in place of $\mathbf{v}_{12}(t)$ for determining $\lambda$, Eq. (41) would have given a rather complicated expression which, however, can be reduced to

$$
\mathbf{r}_{12}(t+h)^{2}-\mathbf{r}_{12}(t)^{2}=\mathbf{r}_{12}(t)^{2}-\mathbf{r}_{12}(t-h)^{2}+O\left(h^{3}\right)+\epsilon_{n}
$$

So by violating time symmetry in the dynamics an additional error term appears. The extra error term is of order $h^{3}$. The dynamics is irreversible (due to the odd power of $h$ ) and the effect of the extra error is disastrous (Sec. IV).

Fortunately it is possible to improve the stability of the dynamics. From Eq. (42) one notices that if $\gamma$ in Eq. (19) or Eq. (35) is replaced by

$$
\begin{aligned}
\gamma_{1}= & \gamma+\left(\mu / \mathbf{r}_{12}^{2}(t)\right)\left(\mathbf{v}_{12}(t-h / 2)\right. \\
& \left.\cdot \mathbf{r}_{12}(t) / h-\mathbf{v}_{12}(t-h / 2)^{2} / 2\right),
\end{aligned}
$$

an improved relation is obtained,

$$
\mathbf{r}_{12}(t+h)^{2}=\mathbf{r}_{12}(t)^{2}+\boldsymbol{\epsilon}_{n},
$$

where the drift in the bond length is not carried over at the next time step. We shall call this "method 1." The correction in Eq. (44) to $\lambda$ is zero if the MD has started from a perfect start configuration with $\mathbf{r}_{12}(0)^{2}=\mathbf{r}_{12}(-h)^{2}=d^{2}$ and without any roundoff errors; the correction therefore just ensures that the roundoff errors do not accumulate. The (recursion) relation Eq. (45) has the statistical properties of a random walk. If one again assumes that the errors introduced at different steps of the method are independently distributed stochastic variables, one finds that the variance of the total error in creases with $n$. If the individual errors have a bias, the total bias will also increase with $n$.

One could also aim for ("method 2"),

$$
\mathbf{r}_{12}(t+h)^{2}=\mathbf{r}_{12}(t-h)^{2}+\epsilon_{n},
$$

which is obtained using

$$
\begin{gathered}
\gamma_{2}=\gamma+\left(2 \mu / \mathbf{r}_{12}(t)\right)^{2}\left(\mathbf{v}_{12}(t-h / 2)\right. \\
\left.\cdot \mathbf{r}_{12}(t) / h-\mathbf{v}_{12}(t-h / 2)^{2} / 2\right) .
\end{gathered}
$$

Again this correction is zero if one starts with a perfect start configuration. Since the steps in Eq. (46) are twice as long as in Eq. (45), although the increase in both variance and bias is still linear in $n$, the rate of increase is halved. The price paid is that Eq. (46) sustains an odd-even oscillation and that $\lambda_{2}$ contains $2 \mathbf{v}_{12}(t-h / 2) \cdot \mathbf{r}_{12}(t) / h$ as possible dominating term. Since $2 \mathbf{v}_{12}(t-h / 2)$ is almost orthogonal to $\mathbf{r}_{12}(t)$ for small values of $h$, this term has a relative large roundoff error.

Methods 0,1 , and 2 give the same result for $\mathbf{r}_{12}(t+h)^{2}$ if $\mathbf{r}_{12}(t)^{2}=\mathbf{r}_{12}(t-h)^{2}$. They only differ in the way they handle previously introduced numerical errors. In particular, one notices that at the first step both method 0 and method 2 depend on $\mathbf{r}_{12}(-h)$, which is indirectly given as $\mathbf{r}_{12}(0)-h \mathbf{v}_{12}(-h / 2)$. If $\left|\mathbf{r}_{12}(0)-h \mathbf{v}_{12}(-h / 2)\right| \neq d$ this introduces an error at step 1. This problem can be solved by always using method 1 for the first step.

It is possible to improve the numerical stability further. This can be seen from the (recursion) relation

$$
\mathbf{r}_{12}(t+h)^{2}=\delta \mathbf{r}_{12}(t)^{2}+(1-\delta) d^{2}+\epsilon_{n},
$$

where $|\delta|<1$. If there were no previous errors, the result would be the same as for methods 0,1 , and 2 . The closer $\delta$ is to 0 , the better is the stability. The optimal choice is $\delta=0$, which is achieved from Eq. (44) with ("method 1a"),

$$
\gamma_{1 a}=\gamma_{1}+\frac{\mu\left(\mathbf{r}_{12}(t)^{2}-d^{2}\right)}{2 h^{2} \mathbf{r}_{12}(t)^{2}} .
$$

Since $\mathbf{r}_{12}(t)^{2}-d^{2}=\left(r_{12}(t)+d\right)\left(r_{12}(t)-d\right)$ one can consider the extra contribution to $\lambda$ as a restoring harmonic force with a force constant, which is chosen such that the system reaches the point of equilibrium in one time step $h$. A larger value of $\delta$ corresponds to a smaller force constant. A negative value of $\delta$ gives a larger force constant.

One may, of course, consider the introduction of this extra term as just another form of rescaling. However, here the rescaling is introduced as an integrated part of the method, not as a deus ex machine, necessitated by deficiencies of the original method. Also, we are able to make the rescaling simultaneous with calculating the next step by the integrating algorithm with no additional computational effort. Finally by making the rescaling at each step one avoids errors to grow large.

If one uses Eqs. (28)-(35) for a NVT system with bond constraints instead of Eqs. (15)-(19), then one still gets Eq. (42). Again, one can improve the result by making the same changes to $\gamma(\xi)$ in Eq. (35) as before.

In conclusion, all of the methods $0,1,1 \mathrm{a}$, and 2 work with and without a thermostat and without any appreciable increase in computer time. For a perfect start configuration and without roundoff errors all four methods degenerate to method 0 (Secs. II A and II B). The extended methods ensure numerical stability of the time-reversible discrete MD. Method 0 can be characterized as "the Verlet algorithm for discrete dynamics with holonomic constraints" and the extended methods as the numerical stable versions. 


\section{COUPLED HOLONOMIC CONSTRAINTS}

Section II showed how numerically stable MD algorithms can be derived for a simple constrained dimer system. It is also natural to consider how the algorithms handle molecules with connected holonomic constraints.

For each holonomic constrained distance $d_{i j}$ for a particle pair $(i, j)$, a Lagrange multiplier $\lambda_{i j}$ is introduced in the equations of motion for particles $i$ and $j$ in the same manner as for the dimer system with constraint force $\pm \lambda_{i j} \mathbf{r}_{i j}(t)$. For $n$ multipliers (i.e., $n$ holonomic constraints) one needs $n$ equations. These equations are again provided by second-order derivatives with respect to time of the $n$ holonomic constraints

$$
\mathbf{r}_{i j}(t) \cdot \mathbf{a}_{i j}(t)+\mathbf{v}_{i j}^{2}(t)=0 .
$$

The acceleration $\mathbf{a}_{i j}(t)$ and the velocity $\mathbf{v}_{i j}(t+h / 2)$ for any particle pair $(i, j)$ can in general be written as

$$
\begin{aligned}
& \mathbf{a}_{i j}(t)=\hat{\mathbf{f}}_{i j}(t)-\xi(t) \mathbf{v}_{i j}(t)+\mathbf{p}_{i j}(t), \\
& \mathbf{v}_{i j}(t+h / 2)=\frac{1}{\xi_{1}}\left\{\xi_{2} \mathbf{v}_{i j}(t-h / 2)+h \hat{\mathbf{f}}_{i j}(t)+h \mathbf{p}_{i j}(t)\right\},
\end{aligned}
$$

where we, for convenience, have introduced a new vector notation with $\mathbf{p}_{i j}(t)$, which is a linear function of the number of $\lambda$ appearing in the equations of motion for particles $i$ and $j$, and $\xi_{i}$ in Eqs. (51)-(55) are given by Eqs. (29)-(31). Equation (52) corresponds to Eq. (28) and $\mathbf{p}_{12}(t)=\lambda_{12}(t) \mathbf{r}_{12}(t) / \mu$ in the case of the dimer system. Applying once again the time-symmetric Eqs. (14) and (27) for $\mathbf{v}_{i j}(t)$ and substituting everything into Eq. (50) one arrives at the following $n$ equations:

$$
\frac{h^{2}}{2 \xi_{1}^{2}} \mathbf{p}_{i j}^{2}(t)+\mathbf{B}_{i j}(t) \mathbf{p}_{i j}(t)+C_{i j}(t)=0,
$$

where

$$
\mathbf{B}_{i j}(t)=\mathbf{r}_{i j}(t)\left\{1-\frac{\xi h}{2 \xi_{1}}\right\}+\frac{1}{\xi_{1}^{2}}\left\{h \xi_{2} \mathbf{v}_{i j}(t-h / 2)+h^{2} \hat{\mathbf{f}}_{i j}(t)\right\},
$$

$$
\begin{aligned}
C_{i j}(t)= & \mathbf{r}_{i j}(t) \cdot \hat{\mathbf{f}}_{i j}^{\prime}(t)+\frac{1}{\xi_{1}^{2}}\left\{\xi_{3} \mathbf{v}_{i j}^{2}(t-h / 2)+h^{2} \hat{\mathbf{f}}_{i j}^{2}(t) / 2\right. \\
& \left.+\xi_{2} h \hat{\mathbf{f}}_{i j}(t) \cdot \mathbf{v}_{i j}(t-h / 2)\right\} .
\end{aligned}
$$

$\mathbf{B}_{i j}(t)$ and $C_{i j}(t)$ correspond to $\beta$ [Eq. (34)] and $\gamma$ [Eq. (35)]. These are quadratic equations in $\mathbf{p}_{i j}(t)$ and thereby in $\lambda$. If the constrained distances are connected through common particles (as in a molecule) then some (or all) of these quadratic equations will be coupled. There are no general solutions for solving coupled polynomial equations as in the case of coupled linear equations. ${ }^{14}$ Minimization techniques, Newton-Rapson, or their combined methods can be applied, but each method has its limitations. Obeying time symmetry may therefore seem to be a problem. However, as shown in Sec. IV, these equations can be solved iteratively as individual (analytic) quadratic equations by initially setting all the $\lambda$ in the expression for $\mathbf{p}_{i j}(t)$ to zero except $\lambda_{i j}$ (Sec. IV B).

In the general case for $\mathbf{v}_{i j}(t+h / 2)$ the LFA gives

$$
\begin{aligned}
\mathbf{r}_{i j}^{2}(t+h)-\mathbf{r}_{i j}^{2}(t)= & \mathbf{r}_{i j}^{2}(t)-\mathbf{r}_{i j}^{2}(t-h) \\
& +2 h^{2}\left[\frac{h^{2}}{2 \xi_{1}^{2}} \mathbf{p}_{i j}^{2}(t)+\mathbf{B}_{i j}(t) \cdot \mathbf{p}_{i j}(t)+C_{i j}(t)\right] .
\end{aligned}
$$

The numerical corrections can then easily be derived. The term for method 1a is [see Eqs. (44) and (49)]

$$
\begin{aligned}
C_{i j-1 a}(t)= & C_{i j}(t)+\left(\frac{\mathbf{v}_{i j}(t-h / 2) \cdot \mathbf{r}_{i j}(t)}{h}-\frac{\mathbf{v}_{i j}^{2}(t-h / 2)}{2}\right) \\
& +\frac{\mathbf{r}_{i j}^{2}(t)-d_{i j}^{2}}{2 h^{2}},
\end{aligned}
$$

producing the same scheme as for the dimer system, i.e., all constrained distances equal their prescribed values $d_{i j}$.

The above derived equations and the numerical corrections hold for any system no matter the complexity. Derivation of the $\mathbf{p}_{i j}(t)$ vectors for a very large molecule such as a protein can be automated in a program by constructing (a possible sparse) constraint matrix with elements $\pm 1 / \mathrm{m}$, subtracting the rows $i$ and $j$ and then multiplying it with a column vector with all the constraint forces.

\section{MOLECULAR DYNAMICS SIMULATIONS WITH CONSTRAINTS}

The expressions for constrained MD derived from Secs. II and III are used below for a system of dumbbells (model I, Sec. IV A) and a system of particles with four connected constraints (model II, Sec. IV B).

\section{A. A simple dimer system}

Model I (Ref. 15) consists of $N=1000$ dumbbells corresponding to a simple "united atom" model of toluene with a big phenyl group (A) constrained to a small methyl group (B). The model is tested at a high-density and lowtemperature state, $\rho \sigma_{A A}^{3}=0.9316, k T / \epsilon_{A A}=0.4647$, where the fluid is highly viscous. ${ }^{15}$ Lengths, energies, and times are given in units of the particle A; for details see Ref. 16. The simulations started from an equilibrium state with all bead distances equal to $d$. MD was performed in double-precision (DP) as well as single-precision (SP) arithmetic for NVE and $N V T$ simulations. The result of the first test is given in Table I. The second column is the mean difference $\Sigma\left(r_{i, i+1}-d\right) / N$ of the $N$ constrained bond distances after $n$ time steps using the linear expression Eq. (10) with $\mathbf{v}_{12}(t)=\mathbf{v}_{12}(t-h / 2)$. It is not clear to us how people previously solved the problem of the misfit of time between positions and forces at time $t$ $+n h$ and the velocities at time $t+(n-1 / 2) h$. But since the method of Gauss's principle of least constraint in general is presented as being linear in the Lagrange multiplier $\lambda$, it seems that one must have ignored the requirement of time symmetry in connection with the term $\mathbf{v}_{12}(t)^{2}$ that leads to a quadratic expression for $\lambda$. As can be seen from the second column in Table I, the broken time symmetry rapidly leads to 
TABLE I. Mean difference $\langle r\rangle-d$ in the bond lengths for model I after the $n$th step and for $N V E$-MD using Eqs. (10) and (20) and method 1a. Results are shown for DP and SP, respectively.

\begin{tabular}{cccccc}
\hline \hline$n$ steps & Equation (10) (DP) & Equation (20) (DP) & Equation (20) (SP) & $1 \mathrm{a}(\mathrm{DP})$ & $1 \mathrm{a}(\mathrm{SP})$ \\
\hline 1 & $-1.6 \times 10^{-7}$ & $-1.6 \times 10^{-14}$ & $-6.5 \times 10^{-9}$ & $-5.6 \times 10^{-15}$ & $9.6 \times 10^{-9}$ \\
5 & $-2.5 \times 10^{-6}$ & $-1.3 \times 10^{-13}$ & $-8.4 \times 10^{-9}$ & $-5.6 \times 10^{-15}$ & $1.1 \times 10^{-8}$ \\
10 & $-7.4 \times 10^{-6}$ & $-3.8 \times 10^{-13}$ & $-1.2 \times 10^{-8}$ & $-5.2 \times 10^{-15}$ & $1.6 \times 10^{-8}$ \\
100 & $-1.4 \times 10^{-4}$ & $-2.6 \times 10^{-11}$ & $-8.2 \times 10^{-8}$ & $-5.6 \times 10^{-15}$ & $6.4 \times 10^{-9}$ \\
1000 & $-2.0 \times 10^{-3}$ & $-2.5 \times 10^{-9}$ & $-1.0 \times 10^{-5}$ & $-5.8 \times 10^{-15}$ & $1.5 \times 10^{-8}$ \\
10000 & Wrong & $-2.5 \times 10^{-7}$ & $-1.1 \times 10^{-3}$ & $-5.6 \times 10^{-15}$ & $1.4 \times 10^{-8}$ \\
\hline \hline
\end{tabular}

a divergence in the bond distances in accordance with Eq. (43), and the improved extrapolation $\mathbf{v}_{12}(t)=\mathbf{v}_{12}(t-h / 2)$ $+(h / 2) \mathbf{f}_{12}(t)$ does not help. The divergence is not due to roundoff errors but is caused by the broken time symmetry; the data in the next two columns demonstrate this. The third column is obtained in DP from Eq. (20) together with Eqs. (17)-(19) (method 0) for the coefficients in the quadratic equation. Here the small errors are caused by roundoff at the arithmetic operation, but they accumulate according to Eq. (42) and will sooner or later spoil the simulation. Column 4 is the same but in SP. The last two columns are the corresponding data for method $1 \mathrm{a}$, which are stable within machine accuracy.

The second test is performed for $N V T$-MD (Table II) for method 0 and the improved expressions, Eq. (44) (method 1), Eq. (49) (method 1a), and Eq. (47) (method 2). The $N V T$-MD simulations started from the same positions as used for the $N V E$-MD simulations. The first thing to notice is that the inclusion of a thermostat does not destroy the effectiveness of the quadratic expressions for the bond constraint; in fact it seems to improve the algorithm, as can be seen by comparing the third column in Table I with the second column in Table II.

Sooner or later accumulated roundoff errors, however, ruin the simulations (method 0 ) although it is possible to maintain the stability over millions of time steps without any adjustment. The next three columns in Table II show that the algorithms derived from Sec. II C stabilize the simulation to an extent that one can simulate the system over hundreds of millions of time steps. Thus method 1a must be characterized as completely stable. The mean values of the thermostat parameter $\Sigma \xi(i) / n$ of the 16384000 time steps are given in the last lines of Table II. For reversible dynamics at an equilib- rium state this average for a long simulation shall be zero and all the quadratic expressions obey this requirement. In accordance with this fact we notice that there is no drift in the energies for the $N V E$-MD using any of the quadratic and time-reversible methods. The origin of the energy conservation is discussed and illustrated in Sec. V.

The radial distribution functions obtained from the different quadratic expressions are identical within the accuracy of the simulations. Figure 1 shows the distributions together with the radial distribution functions obtained by using the program package GROMACS with the LINCS-constraint method $^{5}$ (the agreement is so good that it is not possible to see any difference in $g(r)$ from a figure).

The mean-square displacements $(\mathrm{msd})$ were determined from 20 sets of $n=2^{14} \times 1000=16384000$ time steps. The diffusion constant $D$ obtained from the diffusive regime is $D=3.0 \pm 0.1 \times 10^{-5}$ and the same for all four methods. The sets for methods 0,1 , and 2 were obtained by using method $1 \mathrm{a}$ as the generator of start configurations for the other methods. The data $[D$ and $\operatorname{msd}(t)]$ were compared to the data obtained from NVT-GROMACS+LINCS, and they agree within the accuracy of the simulations.

The simulations were repeated for a state point $\rho \sigma_{A A}^{3}$ $=0.5, k T / \epsilon_{A A}=1$, which approximately corresponds to room temperature conditions and with the same conclusion that all methods conserve energy (NVE-MD) and the constraint with and without a thermostat and agree with the corresponding data obtained by NVT-GROMACS+LINCS

Constrained dynamics are used in many different contexts. Here we have tested the algorithms on a constrained intramolecular bond length. The main reason for constraining the fast intramolecular modes is that one can integrate the molecular motions over much longer time. Typically one

TABLE II. Mean difference $\langle r\rangle-d$ in the bond lengths after the $n$th time step and for $N V T$-MD. Results are shown for DP and model I; method 1a is also shown for SP. $\xi$ (mean) is the mean value of the friction parameter of the thermostat obtained from the 16384000 time steps.

\begin{tabular}{|c|c|c|c|c|c|}
\hline$n$ steps & Method 0 & Method 1 & Method 1a & Method 2 & Method 1a (SP) \\
\hline 1 & $-9.2 \times 10^{-15}$ & $2.6 \times 10^{-16}$ & $1.1 \times 10^{-16}$ & $1.4 \times 10^{-14}$ & $9.6 \times 10^{-9}$ \\
\hline 5 & $1.3 \times 10^{-14}$ & $2.1 \times 10^{-14}$ & $-7.0 \times 10^{-15}$ & $1.4 \times 10^{-14}$ & $7.1 \times 10^{-9}$ \\
\hline 10 & $6.3 \times 10^{-14}$ & $2.6 \times 10^{-14}$ & $9.3 \times 10^{-15}$ & $2.2 \times 10^{-14}$ & $1.3 \times 10^{-8}$ \\
\hline 100 & $-4.1 \times 10^{-12}$ & $-9.6 \times 10^{-14}$ & $1.3 \times 10^{-14}$ & $-5.9 \times 10^{-14}$ & $3.7 \times 10^{-9}$ \\
\hline 1000 & $1.1 \times 10^{-10}$ & $-6.5 \times 10^{-14}$ & $1.1 \times 10^{-14}$ & $3.7 \times 10^{-14}$ & $1.4 \times 10^{-8}$ \\
\hline 10000 & $5.9 \times 10^{-10}$ & $2.0 \times 10^{-12}$ & $1.1 \times 10^{-14}$ & $6.1 \times 10^{-14}$ & $1.9 \times 10^{-8}$ \\
\hline 16384000 & $-7.4 \times 10^{-4}$ & $-9.9 \times 10^{-12}$ & $1.3 \times 10^{-14}$ & $2.7 \times 10^{-11}$ & $2.8 \times 10^{-9}$ \\
\hline$\xi($ mean $)$ & $-1.4 \times 10^{-7}$ & $-4.3 \times 10^{-8}$ & $-4.5 \times 10^{-7}$ & $-1.6 \times 10^{-7}$ & $9.4 \times 10^{-7}$ \\
\hline
\end{tabular}




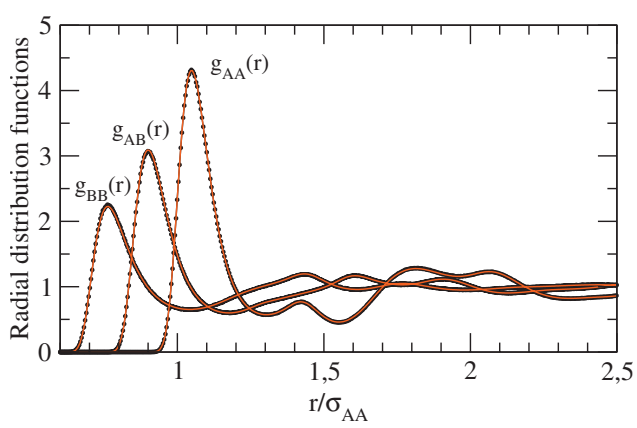

FIG. 1. Radial distribution functions for model I (toluene). Full lines (red) are for the NVT-GROMACS+LINCS and $g_{A B}(r)$ obtained by method 1 are shown with circles. $g_{A A}(r)$ is the phenyl-phenyl distribution, $g_{A B}(r)$ is the phenyl-methyl distribution, and $g_{B B}(r)$ is the methyl-methyl distribution for the dumbbell model of toluene.

needs a time step of order ten times smaller if one wishes to include a constrained mode in the dynamics. The present simulations were mostly performed for a time step $h$ $=0.0025$, which for the toluene model corresponds to a time step $h=4.55 \times 10^{-15} \mathrm{~s}$. The limit of stability was tested by increasing the value of $h$. For $h=0.005$ all four quadratic algorithms were stable with no difference in the obtained data (radial distribution functions and msd). For $h=0.01$ the radial distribution functions were the same as for $h=0.0025$ (Fig. 1), but there were small changes in the msd. All four algorithms failed for $h=0.0125$.

\section{B. A system with complex constrained molecules}

The equations derived from Sec. III are valid for any holonomic constrained molecule. For a system with connected constrained distances one must solve coupled quadratic equations. These equations can be solved as an analytic quadratic equation of one variable keeping all other variables constant. The equations are iterated consecutively by using the previously determined values of $\lambda_{i j}$ at each step, at the start of each time $t$ by

$$
\begin{aligned}
\frac{h^{2}}{2 \xi_{1}^{2}} \mathbf{p}_{12}^{2}(t)+\mathbf{B}_{12}\left(t, \lambda_{i j}(t)\right. & =0, i j \neq 12) \cdot \mathbf{p}_{12}(t)+C_{12}\left(t, \lambda_{i j}(t)\right. \\
& =0, i j \neq 12)=0
\end{aligned}
$$

and then

$$
\begin{aligned}
\frac{h^{2}}{2 \xi_{1}^{2}} \mathbf{p}_{13}^{2}(t)+\mathbf{B}_{13}\left(t, \lambda_{i j}(t)=\right. & 0, i j \neq 12,13) \cdot \mathbf{p}_{13}(t) \\
& +C_{13}\left(t, \lambda_{i j}(t)=0, i j \neq 12,13\right) \\
= & 0
\end{aligned}
$$

etc, and then using the last obtained values for $\lambda_{\alpha \beta}(t)$ in the $n$th iteration for $\lambda_{i j}(t)$. The iteration scheme is used for a system of $N=320$ molecules with four constraints. The molecule is a united atom model of 4-methyl-1,2diphenylbenzene and contains a ring and a branch point with three constraints, one to the methyl group. The angles between the plane of the constrained phenyl groups $(1,2,3)$ and the methyl group (4) are not constrained. The united atom model is shown in Fig. 2, and the result of a NVT-simulation (method 1a) is given in Table III. The consecutive iteration

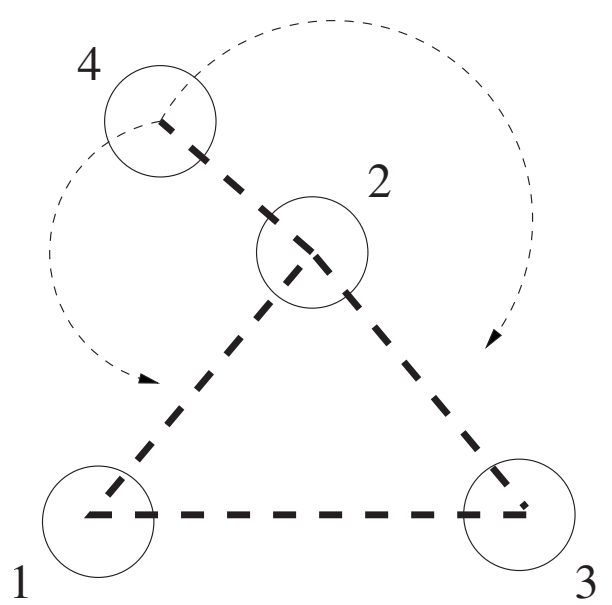

FIG. 2. United atom model for 4-methyl-1,2-diphenylbenzene. The angles between the methyl group (4) and the plane of the phenyl groups are not constrained.

scheme determines the Lagrange multipliers in the order 12 , 13, 23, and 24. The table shows that the last bond is adjusted with SP accuracy already by only one loop at each time step and starting from $\lambda_{i j}(t)=0$. At each time step only eight to ten iteration loops are enough to arrive at a machineprecision conserved bond length for all four connected bonds. The same conclusion (not shown) is reached for a NVE simulation (SP) over $1 \times 10^{6}$ time steps.

To ensure that the algorithm conserves all the bond lengths over long time scales, which is important if one wants, e.g., to study highly viscous liquids, a long SP NVT simulation was conducted. $1 \times 10^{9}$ time steps $(h=0.0025)$ were simulated where the instantaneous root-mean square deviation for all four bonds after the $n=10^{9}$ time step was of the order $O\left(10^{-7}\right)$. This shows that method 1a must be characterized as absolutely stable. It is important to notice that every equation is solved analytically in $O(1)$ time. Hence the runtime of the constraint-force algorithm scales linearly with the number of coupled constraints provided that the number of necessary iteration loops scales slower.

\section{SUMMARY AND DISCUSSION}

Gauss's principle of least constraint introduced into MD in Ref. 2 results in an equation for the Lagrangian multiplier $\lambda$ with a quadratic dependence on the velocities. A timesymmetrical expression used in the (time-)discrete MD for the velocities implies that the time-symmetrical MD with constraints is quadratic in $\lambda$, too. The dynamics is stable over millions of time steps if the time-symmetrical expression [Eq. (20)] is used with no drift in the energy of the NVE-MD simulation (Table I). If on the other hand time symmetry is broken, the linear expression for $\lambda$ introduces a drift in the bond lengths and the NVE simulations fail after only a few hundred time steps. It is not possible to perform proper NVE-MD with the usual bond-scaling procedure. ${ }^{1-3,5,6}$

Analysis of the discrete dynamics (Sec. II C) demonstrates that even when MD is started from a perfect configuration, the roundoff errors introduced per time step accumulate and sooner or later ruin the simulation. But it is possible to modify the Lagrangian multiplier such that that a drift in 
TABLE III. Instantaneous root-mean square deviation $\operatorname{rms}\left(\delta r_{i j}\right)=\sqrt{\sum_{N}\left(r_{i j}-d_{i j}\right)^{2} / N}$ for the $N$ constrained distances after the $n$th time step in $N V T$-MD. The simulated molecule is an isosceles triangle with a side group attached ( $N=320$ molecules) and with four coupled constrained distances (Fig. 2). Results are shown for SP with method 1a $(h=0.0025)$ and Eq. (53) is iterated in the order $\lambda_{12}, \lambda_{13}, \lambda_{23}, \lambda_{24}$.

\begin{tabular}{|c|c|c|c|}
\hline & 1 iteration $(\mathrm{SP})$ & 5 iterations $(\mathrm{SP})$ & 10 iterations (SP) \\
\hline$n$ steps & $\operatorname{rms}\left(\delta r_{12}\right), \operatorname{rms}\left(\delta r_{13}\right), \operatorname{rms}\left(\delta r_{23}\right), \operatorname{rms}\left(\delta r_{24}\right)$ & $\operatorname{rms}\left(\delta r_{12}\right), \operatorname{rms}\left(\delta r_{13}\right), \operatorname{rms}\left(\delta r_{23}\right), \operatorname{rms}\left(\delta r_{24}\right)$ & $\operatorname{rms}\left(\delta r_{12}\right), \operatorname{rms}\left(\delta r_{13}\right), \operatorname{rms}\left(\delta r_{23}\right), \operatorname{rms}\left(\delta r_{24}\right)$ \\
\hline 1 & $2.0 \times 10^{-4}, 1.2 \times 10^{-4}, 1.6 \times 10^{-4}, 1.6 \times 10^{-7}$ & $3.1 \times 10^{-6}, 1.7 \times 10^{-6}, 1.8 \times 10^{-6}, 1.5 \times 10^{-7}$ & $1.8 \times 10^{-7}, 1.8 \times 10^{-7}, 1.9 \times 10^{-7}, 1.6 \times 10^{-7}$ \\
\hline 11 & $2.6 \times 10^{-4}, 1.4 \times 10^{-4}, 1.8 \times 10^{-4}, 1.5 \times 10^{-7}$ & $2.8 \times 10^{-6}, 1.4 \times 10^{-6}, 1.5 \times 10^{-6}, 1.4 \times 10^{-7}$ & $1.7 \times 10^{-7}, 1.9 \times 10^{-7}, 1.9 \times 10^{-7}, 1.6 \times 10^{-7}$ \\
\hline 101 & $2.5 \times 10^{-4}, 1.5 \times 10^{-4}, 2.1 \times 10^{-4}, 1.7 \times 10^{-7}$ & $2.8 \times 10^{-6}, 1.8 \times 10^{-6}, 1.8 \times 10^{-6}, 1.7 \times 10^{-7}$ & $1.8 \times 10^{-7}, 1.8 \times 10^{-7}, 1.8 \times 10^{-7}, 1.5 \times 10^{-7}$ \\
\hline 10001 & $2.5 \times 10^{-4}, 1.7 \times 10^{-4}, 2.4 \times 10^{-4}, 1.6 \times 10^{-7}$ & $3.1 \times 10^{-6}, 2.1 \times 10^{-6}, 2.1 \times 10^{-6}, 1.7 \times 10^{-7}$ & $2.0 \times 10^{-7}, 2.1 \times 10^{-7}, 1.8 \times 10^{-7}, 1.6 \times 10^{-7}$ \\
\hline 1000001 & $2.9 \times 10^{-4}, 1.5 \times 10^{-4}, 2.0 \times 10^{-4}, 1.7 \times 10^{-7}$ & $3.1 \times 10^{-6}, 1.9 \times 10^{-6}, 2.0 \times 10^{-6}, 1.6 \times 10^{-7}$ & $1.8 \times 10^{-7}, 1.9 \times 10^{-7}, 1.9 \times 10^{-7}, 1.6 \times 10^{-7}$ \\
\hline 5000001 & $2.7 \times 10^{-4}, 1.6 \times 10^{-4}, 1.9 \times 10^{-4}, 1.7 \times 10^{-7}$ & $3.0 \times 10^{-6}, 1.6 \times 10^{-6}, 1.7 \times 10^{-6}, 1.7 \times 10^{-7}$ & $2.0 \times 10^{-7}, 1.9 \times 10^{-7}, 1.8 \times 10^{-7}, 1.6 \times 10^{-7}$ \\
\hline $10^{9}$ & & & $1.7 \times 10^{-7}, 2.0 \times 10^{-7}, 1.6 \times 10^{-7}, 1.5 \times 10^{-7}$ \\
\hline
\end{tabular}

the bond length is not carried over to the next time step [Eqs. (44) and (47)]. This stabilizes the NVE-MD as well as the $N V T$-MD (Table II) to a degree where it is possible to perform hundreds of millions of time steps without any adjustments (methods 1 and 2). Finally, it is possible to introduce a restoring bond force directly in the Lagrangian multiplier [Eq. (49)] by which the bond length is conserved within machine accuracy in every step (method 1a). There are no detectable differences in any of the obtained data for the different quadratic methods (radial distribution functions and msd) using either $N V E$ or $N V T$.

Investigations of the $N V E$ stability of MD and using the Verlet algorithm, Eq. (13), for a system of LJ particles show ${ }^{13}$ that there might be a "shadow Hamiltonian" for the system for which the generated discrete positions are exact, and that the time-reversible discrete dynamics therefore contains a "hidden invariance," i.e., the energy which tends to the usual expression for the conserved energy as the discrete dynamics approaches the analytic dynamics, i.e., $h \rightarrow 0$. This result was obtained by deriving exact expressions for the velocities and the kinetic energy for a simple one dimensional harmonic oscillator with central difference discrete dynamics and using these expressions for a three dimensional LJ system. The generated positions were treated "harmonically" by obtaining the instant ("harmonic") amplitudes and frequencies for the individual particles from three consecutive positions per particle during the discrete time evolution. Doing so, the observed variance of the total energy fluctuations decreased significantly. ${ }^{13}$ This indicates the existence of a hidden invariance, the energy for this discrete dynamics. The same hidden invariance is observed for the simple system (model I). (A one dimensional constrained dimer in a harmonic potential obeys the same exact relations as the monomer.) The result of a simulation of the dimer system (model I), where the kinetic part of the energy is sampled in the usual way as $\Sigma_{N} \frac{1}{2} m_{i}\left(\mathbf{r}_{i}(t+h)-\mathbf{r}_{i}(t-h)\right)^{2} / 4 h^{2}$ and in the way derived from Ref. 13, is shown in Fig. 3. We notice that if the kinetic energy is sampled as described in Ref. 13, the standard deviation in the total energy per molecule decreases from $2.23 \times 10^{-5}$ (SP) to $6.94 \times 10^{-6}(\mathrm{SP})$.

In summary we conclude that the derived algorithm, method 0 , is the extension of the usual Verlet algorithm to a system with constraints; this extension has the same behavior and stability as the simple central difference algorithm has no constraints. The modified versions, methods 1,2 , and $1 \mathrm{a}$, degenerate to method 0 when the discrete dynamics starts from a perfect configuration and without any roundoff errors, and the modified versions overcome the numerical instability caused by the roundoff errors, especially method 1a.

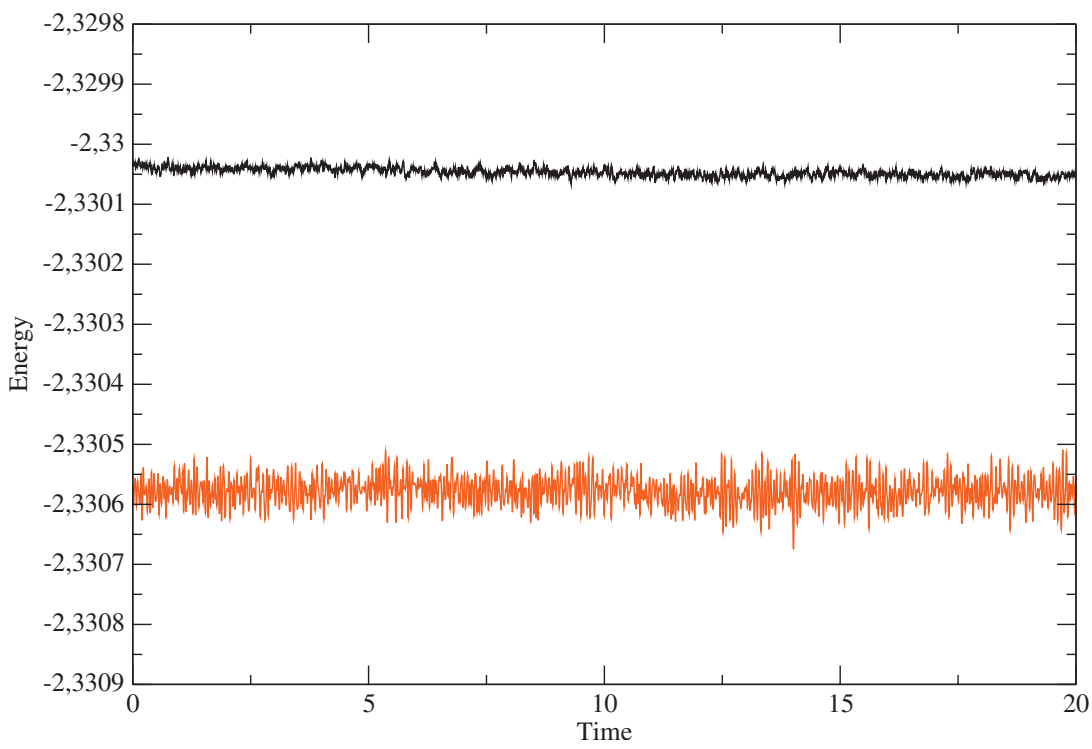

FIG. 3. Energy per particle for the model dimer with constrained distance (method 1a with $h=0.0025$ ), where the two masses of the dimer are equal. The red curve gives the energy per particle for a traditional recording of the kinetic part of the energy and the black curve gives the energy obtained in the way described in Ref. 13. 
The fundamental problem with ensuring time symmetry in the dynamics of molecules with connected constraints is given in Secs. III and IV B. The quadratic expressions complicate MD for systems with connected constraints, but this is easily overcome by simple consecutive iteration loops [Eqs. (58) and (59)]. Constrained MD is heavily used for simulations of complex biosystems with many thousands constraints and at constant pressure and temperature. The fact that the consecutive iteration scheme functions so well implies that it might be possible to use the algorithm in parallel processing as is the case, e.g., for GROMACS with the P-LINCS constraints. ${ }^{17}$ The constrained MD at constant pressure is more complex, ${ }^{18-20}$ and it remains to be demonstrated that one can derive a central-difference barostat algorithm for systems with constraints.

\section{ACKNOWLEDGMENTS}

The center for viscous liquid dynamics Glass and Time is sponsored by the Danish National Research Foundation (DNRF).

${ }^{1}$ J. P. Ryckaert, G. Ciccotti, and H. J. C. Berendsen, J. Comput. Phys. 23, 327 (1977).

${ }^{2}$ R. Edberg, D. J. Evans, and G. P. Morriss, J. Chem. Phys. 84, 6933 (1986).

${ }^{3}$ A. Baranyai and D. J. Evans, Mol. Phys. 70, 53 (1990).
${ }^{4}$ S. W. de Leeuw, J. W. Perram, and H. G. Petersen, J. Stat. Phys. 61, 1203 (1990).

${ }^{5}$ B. Hess, H. Bekker, H. J. C. Berendsen, and J. G. E. M. Fraaije, J. Comput. Chem. 18, 1463 (1997).

${ }^{6}$ S. Toxvaerd, J. Chem. Phys. 107, 5197 (1997).

${ }^{7}$ S. Jang and G. A. Voth, J. Chem. Phys. 107, 9514 (1997).

${ }^{8}$ X. W. Wu and S.-S. Sung, J. Comput. Chem. 19, 1555 (1998).

${ }^{9}$ G. Kalibaeva, M. Ferrario, and G. Ciccotti, Mol. Phys. 101, 765 (2003).

${ }^{10}$ L. Landau and E. Lifshitz, Mechanics (Pergamon, New York, 1961).

${ }^{11}$ L. Verlet, Phys. Rev. 159, 98 (1967).

${ }^{12}$ S. Toxvaerd, Mol. Phys. 72, 159 (1991).

${ }^{13}$ S. Toxvaerd, Phys. Rev. E 50, 2271 (1994).

${ }^{14}$ W. H. Press, S. A. Teukolsky, W. T. Vetterling, and B. P. Flannery, $\mathrm{Nu}-$ merical Recipes, 3rd ed. (Cambridge University Press, Cambridge, England, 2007).

${ }^{15}$ U. R. Pedersen, T. Christensen, T. B. Schrøder, and J. C. Dyre, Phys. Rev. E 77, 011201 (2008).

${ }^{16}$ The dumbbell is a Lennard-Jones dimer model of toluene with the phenyl (A) mass $m_{A}=77.106 u, \sigma_{A, A}=0.4963 \mathrm{~nm}$, and $\epsilon_{A, A} / k=688.68 \mathrm{~K}$. The corresponding parameter values for the methyl group (B) are $m_{B}$ $=15.035 u, \sigma_{B, B}=0.3910 \mathrm{~nm}$, and $\epsilon_{B, B} / k=80.515 \mathrm{~K}$. Bond length $d$ $=0.29 \mathrm{~nm}$. The interactions between unlike subunits are obtained by applying the Lorentz-Berthelot mixing rules. The state point investigated is $(T, \rho)=\left(320 \mathrm{~K}, 1.166 \mathrm{~g} \mathrm{~cm}^{3}\right)$ which can be characterized as a lowtemperature high-pressure state. The time step used, in general, for integrating is $h=0.0025 \approx 4.55 \times 10^{-15}$ s. For details see Ref. 15 .

${ }^{17}$ B. Hess, J. Chem. Theory Comput. 4, 116 (2008).

${ }^{18}$ G. J. Martyna, M. E. Tuckerman, D. J. Tobias, and M. L. Klein, Mol. Phys. 87, 1117 (1996).

${ }^{19}$ M. E. Tuckerman, E. Mundy, and G. J. Martyna, Europhys. Lett. 45, 149 (1999).

${ }^{20}$ G. Ciccotti, G. J. Martyna, S. Melchionna, and M. E. Tuckerman, J. Phys. Chem. B 105, 6710 (2001). 